\title{
Hydrocephalus treated with VP shunt surgery: a clinical audit
}

\author{
Vinod K S Gautam $^{1 *}$, Ravinder Singh ${ }^{2}$, Sarbjeet Khurana ${ }^{3}$ \\ ${ }^{1}$ Department of Neurosurgery Institute of Human Behaviour \& Alled Sciences, Faculty of Medical Science, University of Delhi \\ 2 Department of Medical Anthropology Institute of Human Behaviour \& Alled Sciences, Faculty of Medical Science, University of Delhi \\ ${ }^{3}$ Department of Epidemiology Institute of Human Behaviour \& Alled Sciences, Faculty of Medical Science, University of Delhi \\ *Corresponding author E-mail: medicalanthropology.ihbas@gmail.com
}

\begin{abstract}
Hydrocephalus is a common disease of the brain. Ventriculoperitoneal shunt (VPS) surgery is one of the procedures for its treatment. The clinical outcome following VPS should be evaluated periodically to reduce the complications associated with this procedure. Clinical audit helps to identify and develop effective methods for improvement in patient care. This paper illuminates the experience of authors in clinical audit of forty three neurosurgical patient undergone VPS surgery.
\end{abstract}

Keywords: Clinical Audit, Hydrocephalus, VP Shunt.

\section{Introduction}

The management of hydrocephalus has evolved through the development of various shunting and bypassing operations (Robertson et al. 1973). Shunts drain excess cerebrospinal fluid (CSF) into the peritoneal cavity (ventriculo-peritoneal shunt), right atrium (ventriculo-atrial shunt) and pleural cavity (ventriculopleural shunt) of which ventriculoperitoneal shunt (VPS) surgery is commonest [Low et al.2010; Jha et al.2010). In selected conditions endoscopic third ventriculostomy (ETV) is an alternative to VP shunt surgery. External ventricular drainage (EVD) is a procedure of choice for CSF diversion in cases with progressive post hemorrhagic hydrocephalus and ventriculitis. EVD can also be used as a temporary procedure for reducing intracranial pressure (ICP) during neurosurgical procedure (Weninger et al. 1992, Ali et al. 2013).

Success of VP shunt surgery depends on many factors. The purpose of this clinical audit is to systematically review standard of clinical care of patients. Clinical audit is a quality improvement cycle that involves measurement of the effectiveness of care against agreed and proven standards. The audit cycle includes the assessment of clinical management according to the standards of quality care and interventions then to re-measure the outcome to make further improvements.

\section{Material and methods}

We conducted this study in neurosurgery department of a tertiary care neuropsychiatry hospital in Delhi, India. Forty three patients of hydrocephalus were selected by the non-probability convenience sampling for the study. These were operated between January 2008 and October 2013 by the first author for ventriculoperitoneal shunt placement. Medical charts, operative notes, imaging studies and clinical follow-up evaluations were reviewed and analyzed. Demographic detail, clinical presentation, radiological features, indications for surgery, surgical findings and shunt related complications were analyzed. 16 standards were defined for the comparison for any deviation from the standard protocol for the surgical management of hydrocephalus patients. These standards were based on local guidelines (Fig.1)

Data collected by a team of clinical audit on this particular topic from the case record files of the patients included in study sample. The study was done by the authors who were members of medical audit team of the hospital besides resident doctors.

Table 1: Detail of Standards and Expected Targets

\begin{tabular}{|c|c|c|c|}
\hline $\begin{array}{l}\text { Serial } \\
\text { No }\end{array}$ & Standard & Target & Exceptions \\
\hline 1 & $\begin{array}{l}\text { Detailed pre-op history, physical ex- } \\
\text { amination \& diagnosis }\end{array}$ & 100 & No \\
\hline 2 & Information to the patient & 100 & No \\
\hline 3 & Written consent & 100 & No \\
\hline 4 & $\begin{array}{l}\text { Was the procedure carried out by the } \\
\text { trained Neurosurgeon? }\end{array}$ & 100 & No \\
\hline 5 & CT scan/MRI scan done & 100 & No \\
\hline 6 & $\begin{array}{l}\text { PAC (Pre-anesthetic care) prior to } \\
\text { surgery) }\end{array}$ & 100 & No \\
\hline 7 & Pre-op antibiotic prophylaxis given & 100 & No \\
\hline 8 & $\begin{array}{l}\text { Pre-operative check-list signed by } \\
\text { nursing staff }\end{array}$ & 100 & No \\
\hline 9 & Neuroanesthesia consent form signed & 100 & No \\
\hline 10 & $\begin{array}{l}\text { Record of the monitoring of the patient } \\
\text { during surgery by anesthetist }\end{array}$ & 100 & No \\
\hline 11 & $\begin{array}{l}\text { Operative notes of the neurosurgical } \\
\text { team }\end{array}$ & 100 & No \\
\hline 12 & $\begin{array}{l}\text { Post-op instructions mentioned by the } \\
\text { neurosurgical team }\end{array}$ & 100 & No \\
\hline 13 & $\begin{array}{l}\text { Record of any complications including } \\
\text { Death }\end{array}$ & 100 & $\begin{array}{l}\text { Uneventful } \\
\text { post op }\end{array}$ \\
\hline 14 & $\begin{array}{l}\text { Antibiotic following surgery according } \\
\text { to antibiotic policy }\end{array}$ & 100 & No \\
\hline 15 & $\begin{array}{l}\text { Detailed discharge summary given to } \\
\text { the patient }\end{array}$ & 100 & Death \\
\hline 16 & $\begin{array}{l}\text { Instructions given to the patient to } \\
\text { contact doctor in case of emergency }\end{array}$ & 100 & Death \\
\hline
\end{tabular}




\section{Result}

Data were compiled and analyzed in MS Excel. The statistical output was represented as frequencies and percentages. The tables representing the Frequency and percentage of the diagnosis, surgical procedures and finding were summarized. There was a provision of re-audit within 6 months.

\section{Description of the standards}

Treatment protocol and guideline for the management of a patient with hydrocephalus includes initial assessment with initial assessment comprising of detailed history and examination at the time of admission of the patient, and reassessment of patient and confirmation of hydrocephalus with radiological investigations. The different treatment options including conservative treatment are considered based on the provisional diagnosis of the patient. Surgical options include ventriculoperitoneal shunting, external ventricular drain, and endoscopic third ventriculostomy (ETV). When surgical treatment is planned, pre-anesthetic checkup (PAC) is done by neuroanesthetist. Prior information of surgery is conveyed to all the members of the surgical team, neuroanesthetist operation theater Nurse and other OT staff. Informed consent is taken from the patient and caregiver. Pre-operative instructions are mentioned in the case record file, instructions are verified through Preoperative checklist and then patient is shifted to OT. Third generation injectable cephalosporin is routinely given to all the patients as prophylactic antibiotic at the time of induction of anesthesia. Intraoperative monitoring includes bispectral index (BIS) monitor, temperature, vital signs, capnogram, ECG, central venous pressure (CVP), intraarterial or noninvasive blood pressure monitoring. Ventriculostomy is done to assess of intracranial pressure and collect CSF for investigations. Detailed OT notes and post op instructions and post op neurological assessment are recorded in the case record file.

We have learned through our own experiences in these cases that there were sixteen parameters required for the holistic and evidenced based patients care. The description of these has been divided into three group's viz.i. Standards of pre-operative work-up; ii. Standards at the time of surgery and iii. Post-operative standards

\subsection{Standards of pre-operative work-up}

i) Detailed history, physical examination \& diagnosis: All the patients had undergone detailed clinical work up including history, examination and physical examination by the neurosurgeon. All the patients were investigated systematically through various diagnostic tests including blood profile with complete blood count. Cerebrospinal fluid examination comprising of the entire battery of biochemistry (proteins, sugar), cytology, gram stain , AFB stain, India ink stain and other investigations depending upon provisional diagnosis of the patients. This standard was achievable in 40 cases and in 3 cases the completion of the record was not evident.

ii) Information to the patient: All the patients were informed about the procedure. In 4 cases of total 43 cases the case record file did not contain the evidence that patient had received the detailed information about the surgical procedure and outcome of intervention.

iii) Written consent: An informed written consent was obtained from all the patients prior to surgery. In one case the consent form was signed by neither patient nor operating neurosurgeon. Neurosurgical procedure was done as per the evidence based practice. Hence, the standard which explores that whether the procedure was carried out by the trained neurosurgeon or not? In our cases it was qualified neurosurgeon.

iv) CT scan/MRI scan done: All the patients were diagnosed with radiological investigations like CT scan or MRI of the brain.

\subsection{Standards at the time of surgery}

i) PAC (Pre-anesthetic care prior to surgery): Detailed pre anesthetic check-up was done in all the cases before surgery.

ii) Pre-op antibiotic prophylaxis given: In 25 cases the record of the administration of prophylactic antibiotic was not evident. The main reason for this deficit was due to lack of inclusion of prophylactic antibiotic in printed proformas of anesthesist and neurosurgeon.

iii) Pre-operative check-list signed by nursing staff. In one case the pre-operative checklist was not signed by the nursing staff.

iv) Neuroanesthesia consent form: In two cases the neuroanesthesia consent forms were not signed by the neuroanesthetist.

v) Record of the monitoring of the patient during surgery by Anaesthetist: In one case the intraoperative monitoring was not recorded on the anesthesia record sheet.

vi) Operative notes of the neurosurgical team: In three cases the neurosurgical team did not record the operative notes in detail.

\subsection{Post-operative standards}

i) Post-operative instructions mentioned by the neurosurgical team: In two cases the post-operative instruction were missing in the case record file.

ii) Record of any complications including Death: In all the cases the chronological record of the clinical status were mentioned including the post-operative complications.

iii) Antibiotic following surgery according to antibiotic policy. All the patients received antibiotic according to the antibiotic policy of the Institute.

iv) Detailed discharge summary given to the patient: All the discharged patients received detailed discharge summary with instructions for follow up.

v) Instructions given to the patient to contact doctor in case of emergency: After neurosurgical intervention the patients were assessed in the follow up both radiologically and clinically. In six cases the instructions regarding the person to be contacted in case of emergency was not clearly mentioned.

vi) Results demonstrate deficiencies in documentation which can significantly be improved upon in all standards following the introduction of the standardized proforma.

\section{Discussion}

A total of 43 patients were included in the study. Age of the patients ranged from 4.5 months to 80 years. There were 23 male and 20 female patients. Detailed clinical assessment, preanesthetic checkup, hematological investigations and chest X-ray (posteroanterior view) were done in all patients. Cranial imaging investigations included Ultrasound, CT scan with or without contrast and MRI of the brain with or without contrast and MR spectroscopy. The common etiologies include post meningitic hydrocephalus (48.8\%), congenital hydrocephalus (11.6\%), NPH (9.3\%), Posterior fossa tuberculomas (9.3\%), Neurocysticercus $(6.9 \%)$, \& Posterior fossa tumor $(4.4 \%)$. Other causes were idiopathic, epidermoid, arachnoid cyst and trauma (Table 1). Per operative Ventricular tap was done to assess the depth of the ventricle from the cortical surface and the CSF pressure. CSF was collected in all the cases for cytology and biochemistry and other investigations depending upon the provisional diagnosis of the case. Right sided ventriculoperitoneal shunt with medium pressure valve system was the commonest modality of treatment. All four patients with normal pressure hydrocephalus were treated with low pressure right VP Shunt. One patient of idiopathic hydrocephalus had shown radiologic features of asymmetric hydrocephalus with marked left lateral ventricular enlargement. In this case left sided VP shunt medium pressure was done (Table-2). Two patients died during follow up due to non-surgical reasons. None of 
the patients had any incidence of shunt infection. Shunt related complications included chronic subdural hematoma in 2 cases, per rectal extrusion of peritoneal end in one case. One patient required shunt revision due to malpositioning of the ventricular end.

Table 2: Diagnostic Profile of 43 Cases of Hydrocephalus

\begin{tabular}{lll}
\hline Diagnosis & No of Cases & \% of Total \\
\hline Post Meningitic hydrocephalus & 21 & 48.8 \\
Congenital Hydrocephalus & 5 & 11.6 \\
Normal Pressure Hydrocephalus & 4 & 9.3 \\
Tuberculomas & 4 & 9.3 \\
Neurocysticercosis & 3 & 6.9 \\
Posterior Fossa Tumor & 2 & 4.4 \\
Arachnoid Cyst & 1 & 2.3 \\
Frontal Epidermoid & 1 & 2.3 \\
Trauma & 1 & 2.3 \\
Idiopathic & 1 & 2.3 \\
\hline
\end{tabular}

Table 3: Type of VP Shunt Implanted In 43 Cases of Hydrocephalus Type of shunt implantation No of Cases \% of Total Medium Pressure Right VP Shunt $\quad 38 \quad 88.3$

$\begin{array}{lll}\text { Low Pressure Right VP Shunt } & 4 & 9.3\end{array}$

Medium Pressure Left VP Shunt $\quad 1 \quad 2.3$

The diagnosis of hydrocephalus is based on clinical features, radiologic appearances, and occasionally intracranial pressure recordings. Ultrasonography is the screening procedure in small children when the anterior fontanel is still open. CT scan brain and MRI brain are the preferred method in the detection of parenchymal lesions and in revealing the etiology of the hydrocephalus. Generally, incidence is equal in males and females. NPH has a slight male preponderance. Incidence of hydrocephalus presents a bimodal age curve. One peak occurs in infancy and is related to the various forms of congenital malformations. Another peak occurs in adulthood, mostly resulting from NPH. Adult hydrocephalus represents approximately $40 \%$ of total cases of hydrocephalus (Jha et al. 2010). In our series, post meningitis hydrocephalus was the commonest cause of hydrocephalus $(48 \%)$ followed by congenital hydrocephalus (11\%).

The treatment of hydrocephalus is a challenging one (Low et al. 2010) In untreated hydrocephalus, death may occur by tonsilla herniation secondary to raised ICP with compression of the brain stem and subsequent respiratory arrest. Poor development of cognitive function in infants and children, or loss of cognitive function in adults can complicate untreated hydrocephalus. Visual loss can complicate untreated hydrocephalus and may persist after treatment ( Jha et al. 2010)

Some cases of hydrocephalus can be treated with short term medical treatment with carbonic anhydrase inhibitors, loop diuretic agents, osmotic agents and fibrinolytic therapy. The most suitable drug seems to be acetazolamide, alone or in combination with furosemide. Fibrinolytic therapy administered directly into the ventricular system in cases of post hemorrhagic hydrocephalus(Poca \& Sahuquillo, 2005).Ventricular tapping, repeated lumbar punctures, subcutaneous reservoirs are others measures to treat hydrocephalus. Surgical treatment is the preferred therapeutic option (Jha et al. 2010; Hamilton, 2009). The commonly used surgical procedures are ventriculoperitoneal shunt, endoscopic third ventriculostomy and external ventricular drainage.

The selection of shunt procedure for a patient depends on clinical features and radiological investigations. The right sided shunt surgery is commonly done and in some case left side VP shunt is inserted depending upon the diagnosis. For example, if patient has right sided cerebellopontine angle tumor and right sided retromastoid craniectomy is planned for excisison of the tumor, then in this case left VP shunt is done for treating hydrocephalus. Similarly, in a patient with obstructive hydrocephalus with left sided lateral ventricular enlargement, left VP shunt is done. Usually in patients with obstructive hydrocephalus due to meningitis ventricular tap is done to send CSF for cytology, biochemistry and other investigations depending upon provisional diagnosis. If CSF investigations reveal infection, the external ventricular drain is inserted. If CSF study does not show any feature of meningitis, then VP shunt is done. Type of pressure of the valve of the shunt is decided on the basis of CSF pressure on ventricular tap. Low pressure VP shunt should not be done in children with obstructive hydrocephalus due to aqueductal stenosis. It can lead to overdrainage and subdural hematoma. So, for these patients with congenital hydrocephalus, a medium pressure right VP shunt is most appropriate. In patients of NPH (Normal Pressure Hydrocephalus), low pressure VP shunt is done.

Complications of the shunt can be avoided by taking simple measures like preoperative part preparation, draping, prophylactic antibiotic, minimizing movements of many persons inside the operation theater, choosing appropriate type of the shunt, securing shunt assembly of the shunts, collection of the CSF during the surgery, minimal handling of the shunt assembly, changing of the glove prior to the handling of shunt, antibiotic therapy after insertion of shunt, etc. If every stage of shunt surgery is given due importance, the frequency of shunt revision can be minimized. Once failure is suspected, patient should again be investigated thoroughly before operating again. As long as the ventricular tip is inside the ventricle it is very unlikely that shunt is not functioning. Shunt should not be revised if the ventricular end of the shunt has crossed to the opposite side lateral ventricle piercing septum pellucidum.

The audit cycle includes taking action to bring practice in line with these standards to improve the quality of care and health outcomes. By following the cycle, any clinician or team should be able to see where a particular area or step in their practice which can be improved.

\section{Conclusion}

Ventriculoperitoneal shunting is the most widely used neurosurgical procedure for the management of hydrocephalus. Endoscopic third ventriculostomy is another alternative to treat obstructive hydrocephalus. The high incidence of shunt failure can be minimized by meticulous clinical work up, CSF study, selection of appropriate type of shunt, use prophylactic antibiotic, and precautions at the time of surgery. Shunt surgery is very often performed by the neurosurgical trainees and younger neurosurgeons. This procedure is simple but associated with high failure rate. The review of clinical outcome provides an opportunity for clinical team to rectify and modify the steps of the clinical care and improve the documentation. Re-audit following improved quality and content of the documentation and implementation of standard operating procedure is an effective method for identifying deficits in the clinical management and improves clinical outcome.

Disclaimer: Clinical Audit only needs ethical approval if the audit involves anything being done with patients which would not otherwise be part of their routine clinical management. It is self-audit of the quality of care provided by a single neurosurgeon and it is not related to the work of other clinicians. Audit is carried by the members of hospital medical record audit team, allied sciences professional and resident doctors. 


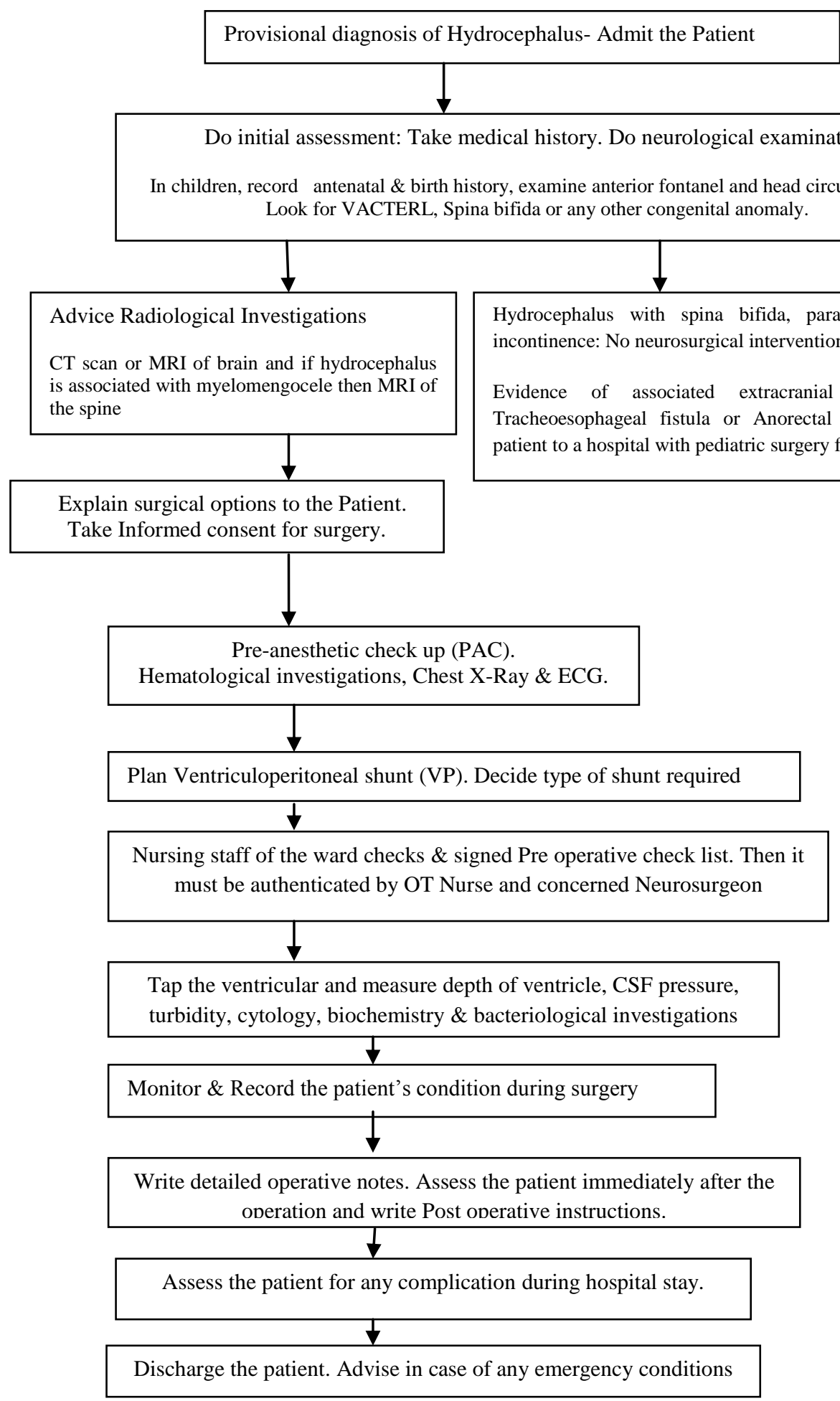

Fig. 1: Flow Diagram for Managing Hydrocephalus

\section{References}

[1] Robertson J.S., Maraqa M.I., Jennett Bryan: Ventriculopperitoneal shunting for hydrocephalus. British Medical Journal, 1973,2, 289292.

[2] Low D, Drake JM, Seow WT, Ng WH. Management of ventriculoperitoneal shunts in the paediatric population.Asian J Neurosurg. 2010 Jan; 5(1):7-14

[3] Jha R, Khadka N, Kumar P. Management of Hydrocephalus - Our Experience.PMJN, Vol 10, Number 2, Jul-Dec 2010, 68-70.

[4] Ali M, Usman M, Khan Z, Khan K.M.,Hussain R,and Khanzada K: Endoscopic third ventriculostomy for obstructive hydrocephalus. Journal of the College of Physicians and Surgeons, Pakistan 2013, Vol. 23(5): 338-341.
[5] Weninger M, Salzer HR, Pollak A, Rosenkranz M, Vorkapic P, Korn A, Lesigang C. External ventricular drainage for treatment of rapidly progressive posthemorrhagic hydrocephalus. Neurosurgery. 1992 Jul; 31(1):52-7; discussion 57-8

[6] Poca MA, Sahuquillo J. Short-term medical management of hydrocephalus. Expert Opin Pharmacother. 2005 Aug; 6(9):1525-38.

[7] Hamilton MG. Treatment of hydrocephalus in adults. Semin Pediatr Neurol. Mar 2009; 16(1):34-41.

[8] Rehman AU, Rehman TU, Bashir HH, Gupta V. A simple method to reduce infection of ventriculoperitoneal shunts. J Neurosurg Pediatr. 2010 Jun;5(6):569-72 